UMR 5824

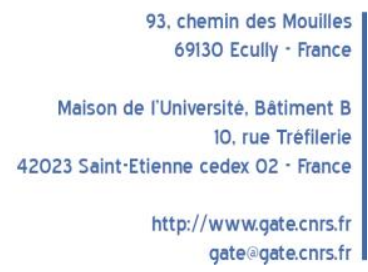

WP 1734 - December 2017

\title{
Information Order Shifts Criterion Placement in Perceptual Decisions
}

\author{
Thomas Garcia, Ismaël Rafaï, Sébastien Massoni
}

\begin{abstract}
:
Facing perceptual decisions with asymmetric stakes, individuals exhibit a conservative criterion placement. This bias prevents them from reaching the optimal decision process defined as the one which maximizes their expected payoffs. We propose in the present experimental study a non-invasive method to correct behaviors toward more optimality. We manipulate the information order between payoff information and perceptual evidence for three different incentive levels invariant regarding Signal Detection Theory predictions. Our results support the effectiveness of such manipulation: the decision strategy shifts toward optimality when payoff information is displayed last. The shift toward optimality is more pronounced for higher payoff contrasts. These results, which cannot be explained within the Signal Detection Theory framework, give new insights on the cognitive processes responsible for the conservative criterion placement
\end{abstract}

\section{Keywords:}

conservative criterion placement, signal detection theory, optimalityaccuracy, information order

JEL codes:

D81, D91, Y8

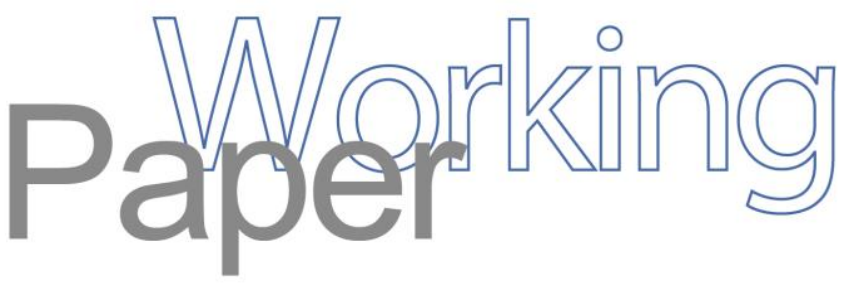




\title{
Information Order Shifts Criterion Placement in Perceptual
}

\section{Decisions}

\author{
Thomas Garcia ${ }^{1,2 *}$ Ismaël Rafai ${ }^{3 \dagger}$ and Sébastien Massoni ${ }^{2,4 \ddagger}$ \\ ${ }^{1}$ Univ Lyon, Université Lumière Lyon 2, GATE UMR 5824, \\ F-69130 Ecully, France \\ ${ }^{2}$ QuBE - School of Economics and Finance, QUT, Brisbane, Australia \\ ${ }^{3}$ GREDEG, Université Côte d'Azur, Nice, France \\ ${ }^{4}$ Australian Centre for Entrepreneurship Research, QUT, Brisbane, Australia
}

December 4, 2017

\begin{abstract}
Facing perceptual decisions with asymmetric stakes, individuals exhibit a conservative criterion placement. This bias prevents them from reaching the optimal decision process defined as the one which maximizes their expected payoffs. We propose in the present experimental study a non-invasive method to correct behaviors toward more optimality. We manipulate the information order between payoff information and perceptual evidence for three different incentive levels invariant regarding Signal Detection Theory predictions. Our results support the effectiveness of such manipulation: the decision strategy shifts toward optimality when payoff information is displayed last. The shift toward optimality is more pronounced for higher payoff contrasts. These results, which cannot be explained within the Signal Detection Theory framework, give new insights on the cognitive processes responsible for the conservative criterion

*garcia@gate.cnrs.fr, 93 Chemin des Mouilles, 69130 Ecully

${ }^{\dagger}$ ismael.rafai@unice.fr, 28 Avenue Valrose, 06100 Nice

${ }^{\ddagger}$ sebastien.massoni@gmail.com, 2 George St, Brisbane City QLD 4000, Australia
\end{abstract}


placement.

Keywords: conservative criterion placement, signal detection theory, optimalityaccuracy, information order.

\section{Introduction}

In perceptual decisions, individuals' choices are guided by stimuli information and decisions' rewards. Stimuli provide evidence on the correct answer while rewards provide the associated consequences. Maximizing accuracy or expected rewards (being optimal) leads to the same behavior if reward differentials between successes and errors are constant across decisions. However, introducing asymmetric rewards implies a divergence in behavior. Reaching accuracy is no more equivalent to being optimal and individuals achieve optimality at the expense of accuracy. Indeed if some decisions lead to higher additional rewards than others, individuals have to concede a lower accuracy for the low rewarded decisions compared to the highly rewarded ones. It ultimately results in an accuracy loss at the benefit of maximal payoffs. As an example consider a physician facing a patient exhibiting ambiguous symptoms that can be related either to a serious or a minor disease. Correctly identifying that the patient suffers from the serious disease is associated with higher stakes than correctly classifying the disease as minor. To maximize her patient's expected welfare, the physician should sometimes diagnose the major disease even if the minor one is more likely.

A robust finding is that facing perceptual decisions most individuals adopt a decision strategy leading to over-accurate but sub-optimal answers (Pitz and Downing, 1967; Maddox and Bohil, 1998; Bohil and Maddox, 2001; Garcia and Massoni, 2017). This bias toward accuracy is referred as "conservative criterion placement". ${ }^{1}$ Several studies have investigated the origins of this bias. Most explanations rely on a suboptimal learning of the

\footnotetext{
${ }^{1}$ This paper focuses on asymmetry induced by payoffs. The conservative criterion placement has also been highlighted for asymmetry in prior probabilities that each answer is correct (base-rate; Ell et al., 2009; Bohil and Wismer, 2015).
} 
criterion placement (Busemeyer and Myung, 1992; Erev, 1998; Maddox and Bohil, 2004). Understanding the origin of the bias is essential, however it is also necessary to find means to correct it. The only studies investigating solutions for decreasing the bias are based on feedback manipulations. Maddox and Dodd (2001), Maddox and Bohil (2005) and Bohil and Wismer (2015) obtain that providing subjects with the optimal classifier's decisions shifts decisions toward optimality. Bohil et al. (2015) obtain similar results by disclosing to subjects the decisions of the best performer of a previous experimental session. Deceptive feedback identifying as correct some wrong decisions also shift decisions toward optimality (Han and Dobbins, 2008). These feedback manipulations require either information on a best classifier or deception on the outcome of the decision. We experimentally investigate a new method to induce more optimal decisions that does not require these constraints. In the present study we manipulate the order in which information used to make a perceptual decision is displayed.

To make their decisions, individuals have two types of information available: information on the likelihood that each decision is correct and information on payoff. Information on the likelihood is extracted from a visual stimulus while payoff information is given by a payoff matrix. In all previous studies, stimuli are given immediately before the decision. Additionally, the payoff matrix is fixed for series of consecutive decisions. In our study, we test the impact of the information order by inverting the order in which both types of information are given to subjects. Assuming that the information presented last has more influence on the decision, the fact that stimuli are usually presented just before the decision may play a role in the observed bias. An efficient way to improve decision strategies would thus consist in presenting the consequences of the decision last. It could enable us to reduce the bias without constraining choices, changing the incentives scheme or even changing the amount of information available. To refine the example previously discussed, the order in which information is delivered reflects two possibilities available to the physician. The consequences of each treatment are established by a cost-benefit analysis based on the patient's health history. The likelihood of each disease is obtained 
after having examined the patient. The physician will examine the patient and investigate the consequences of each treatment but the order in which he leads these two tasks is free for him to choose.

Our analyses rely on Signal Detection Theory (SDT hereafter; Green and Swets, 1966). SDT gives a normative benchmark to study perceptual decision-making. It enables to disentangle the impact of stimuli perception - the discrimination - from the impact of the decision strategy - the criterion. We assess the order effect in decisions by comparing discriminations and criteria for the different information orders. Our study also allows us to test for the robustness of SDT predictions. We used three different payoff contrasts: payoff patterns that are invariant regarding SDT predictions but vary regarding payoff differences between successes and errors for signal and noise alternatives. We are interested in studying if these different payoff contrasts have an impact on the subjects' decisions. It extends the work of Bohil and Maddox (2003) who obtain reduced deviations from optimality when multiplying payoffs by a constant factor.

Results confirm departures from theory. In addition to confirming the conservative criterion placement, they highlight a recency effect, i.e. individuals have better decision strategies when payoff information are given last and weakly higher discriminations when

stimuli are displayed last. Moreover, higher payoff contrasts conduct to more optimal decision strategies.

\section{Method}

\section{Stimuli}

We used 2-alternative forced choice (2AFC) stimuli previously used in Massoni et al. (2014) and Fleming et al. (2016). It consisted of a 700ms display of dots in two circles, one with 50 dots and the other with $c$ more dots (Figure 1-A). Subjects evaluated which circle contained the most of dots. $c$ was calibrated at a subject level to control for heterogeneity 
in visual abilities and kept constant during the experiment. ${ }^{2}$

\section{Task and Procedure}

Participants- 58 students from Queensland University of Technology were enrolled in four 1-hour sessions (age: $M=24.89, S D=5.9 ; 45 \%$ were females). They were paid on average AUD $22.8(S D=4.83)$.

Experiment phase - The experiment was a within-subject design consisting of series of individual perceptual decisions organized in 4 blocks of 72 trials. To make a decision, subjects received two different information: stimuli and payoffs. Payoffs were communicated by a payoff matrix providing a matching rule between the four possible decision outcomes and the number of points earned in each case. By combining both information, participants answered either "Left" or "Right". To help subjects in their decision-making, we gave them an accuracy feedback and a point feedback after each trial as well as an additional aggregated feedback every 12 trials. 28 randomly selected decisions were paid (10 points $=A U D$ 1). In the "Stimuli-First" treatment $(\mathrm{SF})$, subjects observed the stimulus before the payoff matrix. In the "Payoff-First" treatment $(\mathrm{PF})$, the order was reversed (Figure 1-C\&D). Payoff matrices were varied between trials while treatments were varied between blocks. ${ }^{3}$

Payoff Matrices - We used a set of $3 \times 2$ payoff matrices that were invariant regarding the optimal decision criterion but varied regarding the payoff contrast (difference between correct and incorrect payoffs). It is composed of "Low" $\left(M_{12}\right)$, "Intermediate" $\left(M_{17}\right)$ and "High" $\left(M_{21}\right)$ payoff contrasts with higher number of points earned for successes either for "Left" or "Right" (see Table 1 and Figure 1-B). Values have been chosen to respect these theoretical constraints while being visually as close as possible and rewarding similarly perfect performances. ${ }^{4}$ We use negative and positive values as previous studies have

${ }^{2}$ Additional information about stimuli and their calibration are in SI.

${ }^{3}$ Treatment, payoff matrices, stimuli sequences and paid decisions were randomly generated. Payoff matrices, target circles frequencies and the number of paid decisions per block were controlled to be equal. Their sequences were the same for all subjects.

${ }^{4} M_{12}$ and $M_{21}$ are based on the exact same digits at the exception of the 0 used only in $M_{12}$. $M_{17}$ conserves a similar pattern with intermediate values. All matrices give the same expected payoff for perfect performance (11 points) but vary regarding expected payoff for random guess (1 
obtained that pure-gain, pure-loss and mixed gain-loss frameworks do not affect criterion placement (Maddox et al., 2003; Garcia and Massoni, 2017).

\begin{tabular}{lcccc}
\hline \hline Payoff Matrix & Success Left & Success Right & Error Left & Error Right \\
\hline Low $\left(M_{12}\right)$ & 12 & 10 & 9 & 9 \\
Intermediate $\left(M_{17}\right)$ & 10 & 12 & 9 & 9 \\
& 17 & 5 & -1 & -1 \\
High $\left(M_{21}\right)$ & 5 & 17 & -1 & -1 \\
& 21 & 1 & -9 & -9 \\
& 1 & 21 & -9 & -9 \\
\hline
\end{tabular}

Table 1: Set of payoff matrices.

\section{Data analysis}

Based on our 2AFC stimuli and the three different payoff contrasts, we specify our model as a Gaussian equal-variance SDT model with signal being "the highly rewarded circle contains more dots". Reporting "signal" thus consists in choosing the highly rewarded alternative. ${ }^{5}$ The optimal decision criterion is $\beta^{*}=\frac{1}{3}$ for all matrices. ${ }^{6}$

Gaussian equal-variance SDT provides a computational model to estimate both subjects' discrimination $\left(d^{\prime}\right)$ and decision criterion $(\beta)$. To evaluate treatments effects, we estimate one couple $\left(\hat{d}^{\prime}, \hat{\beta}\right)$ for each information order at a subject level (Estimation (1)). To assess the impact of each payoff matrix on the decision strategy, we estimated one decision criterion for each combination of matrices and information orders at a subject level. As the stimuli perception does not depend on the payoff matrix, we estimated a single discrimination for each information order. Estimation (2) thus consists in estimating a vector $\left(\hat{d}^{\prime}, \hat{\beta}_{12}, \hat{\beta}_{17}, \hat{\beta}_{21}\right)$ for each information order at a subject level. We compute estimapoint for $M_{21}, 5$ points for $M_{17}$ and 10 points for $M_{12}$ ).

${ }^{5}$ The equivalence between the $2 \mathrm{AFC}$ specification induced by the perceptive task and our framework is detailed in SI.

${ }^{6}$ The optimal decision criterion is given by the ratio between the additional payoff of a success for the "noise" alternative and the additional payoff of a success for the "signal" alternative:

$$
\beta_{21}^{*}=\frac{1-(-9)}{21-(-9)}=\beta_{17}^{*}=\frac{5-(-1)}{17-(-1)}=\beta_{12}^{*}=\frac{10-9}{12-9}=\beta^{*}=\frac{1}{3}
$$




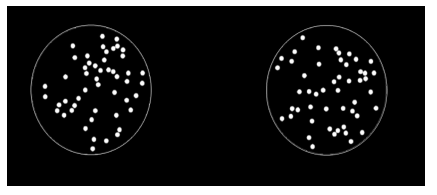

(A) Stimuli (trial dependant)

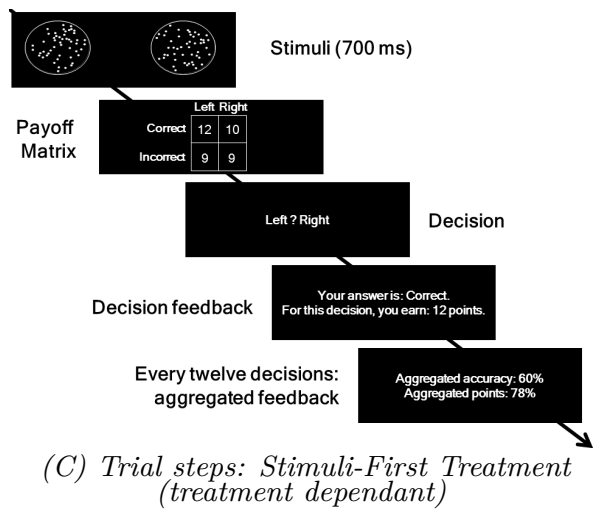

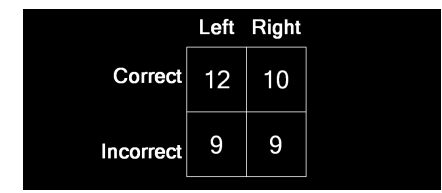

(B) Payoff Matrix (trial dependant)

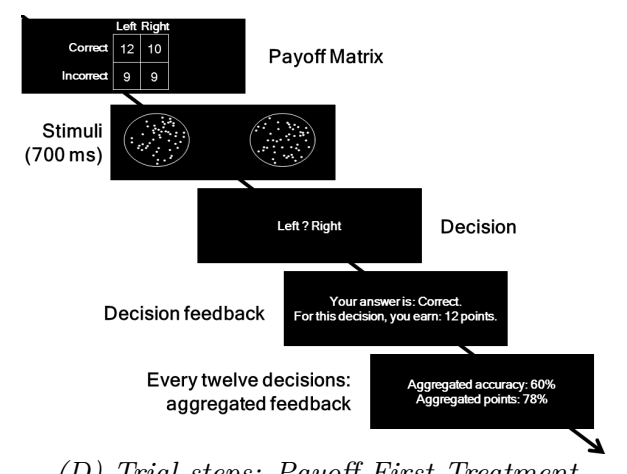

(D) Trial steps: Payoff-First Treatment
(treatment dependant)

Figure 1: Experimental design. (A) Example of stimuli. Subjects evaluated which circle contained the most of dots. (B) Example of a Payoff Matrix. (C) Timeline of a trial for the Stimuli-First Treatment. Facing a fixation cross, subjects initiated the trial. Stimuli appeared for $700 \mathrm{~ms}$ before being replaced by the fixation cross. The payoff matrix was displayed with no time pressure. Subjects made their decision (right or left). Feedback on the accuracy of the answer and the number of points earned was given. Every twelve trials aggregated feedback on the success rate and aggregated points over the last 12 trials was provided. (D) Timeline of a trial for the Payoff-First Treatment. The display order of the payoff matrix and stimuli is reversed compared to the previous timeline. 


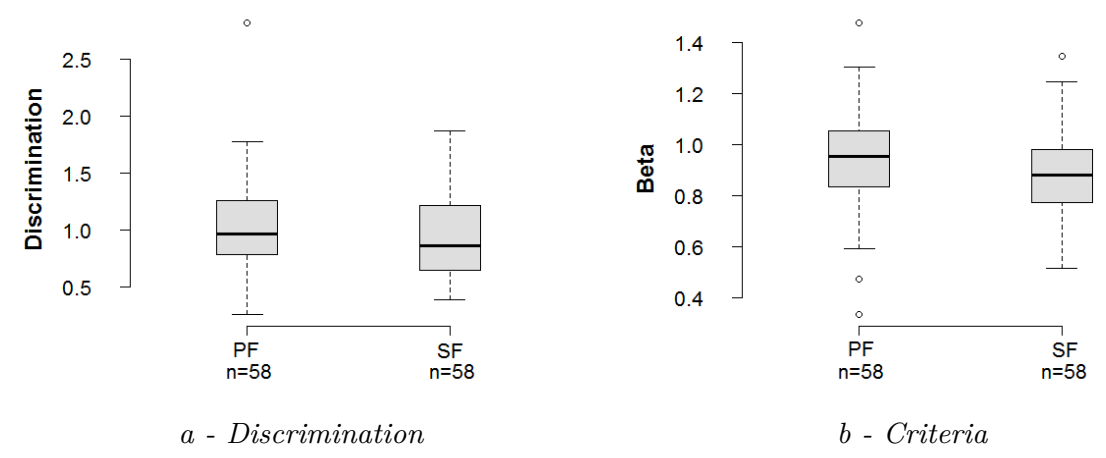

Figure 2: Tukey box-and-whisker plot: Discrimination and criteria for Payoff-First treatment $(\mathrm{PF})$ and Stimuli-First treatment $(\mathrm{SF})$.

tors using maximum likelihood because the analytic form of the estimators is unknown for Estimation (2) and equivalent to the analytic form for Estimation (1) (cf. Ackermann and Landy (2015) for a similar estimation procedure). ${ }^{7}$

Paired t-tests are used to compare two balanced samples. Matrix effects across our 6 samples (3 matrices $\times 2$ treatments) are compared using repeated measures analysis of variance (rANOVA) followed by Tukey's range tests for multiple comparisons of means. We report Cohen's $d_{z}$ and partial Eta-squared as effect size measures.

\section{Results}

\section{Order effect}

Estimated discriminations and criteria for each treatment used to assess the order effect are presented in Figure 2.

Result 1: Subjects use conservative decision criteria.

We observe a mean decision criterion of $0.95(S D=0.202)$ for the Payoff-First treatment and $0.86(S D=0.158)$ for the Stimuli-First treatment. Both decision criteria are lower than the accuracy maximizing decision criterion, $\left(\beta^{a}=1\right),[t(57)=-2.535, p=0.014]$ for $\mathrm{PF}$ and $[t(57)=-5.847, p<0.001]$ for $\mathrm{SF}$; and higher than the optimal decision criterion $\left(\beta^{*}=\frac{1}{3}\right),[t(57)=22.58, p<0.001]$ for PF and $[t(57)=26.31, p<0.001]$ for SF.

\footnotetext{
${ }^{7}$ Estimation methods are detailed in SI.
} 


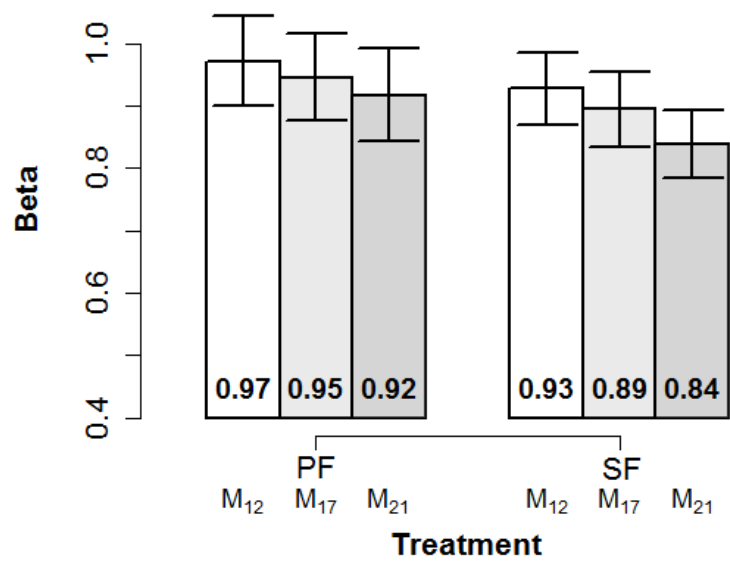

Figure 3: Estimated criteria for each matrix by treatment. $M_{12}, M_{17}$ and $M_{21}$ respectively correspond to "Low", "Intermediate" and "High" payoff contrasts. Error bars correspond to $95 \%$ confidence intervals. Bold figures inside bars are mean values.

Result 2: Subjects exhibit more optimal decision strategies in the Stimuli-First treatment than in the Payoff-First treatment.

Subject in SF exhibit more optimal decision criteria $(M=0.86, S D=0.158)$ than in $\mathrm{PF}(M=0.95, S D=0.202),\left[t(57)=2.280, p=0.026, d_{z}=0.299\right]$. This result is also supported by the rANOVA, $\left[F(1,57)=5.725, p=0.020, \eta_{p}^{2}=0.091\right]$.

Additional result: Subjects exhibit weakly higher discriminations in the Payoff-First treatment.

We find weak evidence that the subjects exhibit a higher discrimination in the PayoffFirst $(M=1.02, S D=0.415)$ compared to the Stimuli-First $(M=0.95, S D=0.391)$ treatment $\left[t(57)=1.854, p=0.069, d_{z}=0.243\right]$.

\section{Payoff Effect}

Estimated criteria for each matrix in each treatment used to assess the matrix effect are presented in Figure 3.

Result 3: Subjects exhibit more optimal decision strategies in presence of higher payoff contrasts.

Decision criteria vary with payoff contrasts $\left[F(2,114)=3.144, p=0.047, \eta_{p}^{2}=0.052\right]$. The presence of the "High" payoff contrast $\left(M_{21}\right)$ leads subjects to adopt a lower decision 
criterion compared to the "Low" payoff contrast $\left(M_{12}\right)$ resulting in more optimal decisions $\left[q=-2.496, p=0.034, d_{z}=0.328\right]$. The criterion associated with the intermediate payoff contrast $\left(M_{17}\right)$ is included between criteria of the two other payoff contrasts. However, it is not statistically different from neither of them $\left[M_{12}: q=-1.044, p=0.549, d_{z}=\right.$ $\left.0.137 ; M_{21}: q=-1.453, p=0.314, d_{z}=0.191\right]$.

\section{Discussion}

We test how the order of presentation between a stimulus and information on payoff affects individuals' discrimination and decision criterion in perceptual decisions for different payoff contrasts. We conclude that displaying payoffs last shifts decision strategies toward optimality and we find weak evidence that receiving visual stimuli last conducts to a higher discrimination. These results give support to a recency effect as displaying a type of evidence last improves its evaluation. Moreover, we find that higher payoff contrasts conduct to more optimal decision strategies. Neither the recency effect nor the contrast effect can be explained within the SDT framework. Indeed, implemented payoff patterns are invariant regarding optimal decision strategies and the order in which individuals receive perceptual and payoff information is not addressed by SDT.

Recency effects have been highlighted in multiple settings as sequential effect in category and probability learning (Jarvik, 1951; Jones and Sieck, 2003), evaluation of contradictory information (Furnham, 1986; Trotman and Wright, 2000) or choice presentation order (Mantonakis et al., 2009; Daniel and Katz, 2017). These types of recency effects have been analyzed in relation with belief updating (Hogarth and Einhorn, 1992). However in our experimental paradigm subjects sequentially receive information on two different components of the decision (the likelihood and the criterion). The recency effect is thus to be analyzed outside of the scope of belief updating. Our result is more closely related to order

effects in memory (Murdock Jr, 1962; Tzeng, 1973; Baddeley and Hitch, 1993; Unsworth and Engle, 2007) as the functioning of the working memory and the underlying attention 
allocation process are relevant in explaining our result (Baddeley and Hitch, 1974). Individuals need to process consecutively two complex tasks: stimuli evaluation and payoff maximization. The information processed first is not immediately used and thus needs to be stored in the working memory. When confronted to the second type of information, individuals need to divide their attention between processing new information and maintaining previous evidence (Case, 1985). Two mechanisms with opposite consequences can be identified: a stronger memory decay on the first type of information (Towse and Hitch, 1995) and a decreased attention when processing the second type (Barrouillet et al., 2004). The recency effect obtained indicates that the first mechanism dominates the second one, receiving the information last improves the quality of its treatment at the decision time.

The contrast effect may also be related to attention allocation. Higher payoff contrasts raise incentives to pay attention to the consequences of the choice. More attention toward the payoff matrix would improve the criterion placement, explaining our contrast effect. Bohil and Maddox (2003) obtain similar results that they interpret as being a consequence of different rates of criterion learning. The fact that we observe the same effect while in our design criterion learning effects are less salient suggests that allocation of attention mechanisms may also play a role in their study. ${ }^{8}$ Likewise, the impact of feedback manipulation on criterion placement previously found, may also be understood within this framework. Oriented feedback toward optimal decision may induce a higher attention allocated to the payoff information, improving the criterion placement.

Milkman et al. (2009) claim that "the optimal moment to address the question of how to improve human decision making has arrived" (p.379). This statement seems particularly valid regarding solutions to reduce the conservative criteria placement. As an answer, we investigate the effect of modifying the information order for rewarded two-alternative forced choices. We obtain that, once controlled for the task difficulty, presenting rewards last has a positive impact on decisions. This simple manipulation is non-intrusive and

\footnotetext{
${ }^{8}$ In our experiment subjects made a smaller number of decision (288 against 1920) and payoffs were varied at a decision level rather than at a block level (120 decisions).
} 
does not require extra information on the decision context. It thus exhibits valuable qualities to guide decision-making. We also find that this manipulation weakly affects the discrimination ability of participants. While designing an intervention that corrects the criterion bias without affecting discrimination remains to be done, our information order intervention seems to be the lightest way to shift behaviors and thus appears as a new step toward inducing optimal criterion placement strategies.

\section{Acknowledgments}

The authors report no conflict of interest. The experiment was supported by an internal grant from QuBE - QUT. The authors are grateful to Agnès Festre, Charlotte Saucet and Marie Claire Villeval for insightful comments.

\section{References}

Ackermann, J. F. and Landy, M. S. (2015). Suboptimal decision criteria are predicted by subjectively weighted probabilities and rewards. Attention, Perception, \& Psychophysics, 77(2):638-658.

Baddeley, A. D. and Hitch, G. (1974). Working memory. Psychology of learning and motivation, 8:47-89.

Baddeley, A. D. and Hitch, G. (1993). The recency effect: Implicit learning with explicit retrieval? Memory \& Cognition, 21(2):146-155.

Barrouillet, P., Bernardin, S., and Camos, V. (2004). Time constraints and resource sharing in adults' working memory spans. Journal of Experimental Psychology: General, 133(1):83.

Bohil, C. J. and Maddox, W. T. (2001). Category discriminability, base-rate, and payoff effects in perceptual categorization. Perception $\&$ Psychophysics, 63(2):361-376.

Bohil, C. J. and Maddox, W. T. (2003). On the generality of optimal versus objective 
classifier feedback effects on decision criterion learning in perceptual categorization. Memory \& Cognition, 31(2):181-198.

Bohil, C. J. and Wismer, A. J. (2015). Implicit learning mediates base rate acquisition in perceptual categorization. Psychonomic bulletin \& review, 22(2):586-593.

Bohil, C. J., Wismer, A. J., Schiebel, T. A., and Williams, S. E. (2015). Best-classifier feedback in diagnostic classification training. Journal of Applied Research in Memory and Cognition, 4(4):368 - 373.

Busemeyer, J. R. and Myung, I. J. (1992). An adaptive approach to human decision making: Learning theory, decision theory, and human performance. Journal of Experimental Psychology: General, 121(2):177.

Case, R. (1985). Intellectual development: Birth to adulthood. Academic Pr.

Daniel, T. and Katz, J. (2017). Primacy and recency effects for taste. Journal of experimental psychology. Learning, memory, and cognition.

Ell, S. W., Ing, A. D., and Maddox, W. T. (2009). Criterial noise effects on rule-based category learning: The impact of delayed feedback. Attention, Perception, 83 Psychophysics, 71(6):1263-1275.

Erev, I. (1998). Signal detection by human observers: a cutoff reinforcement learning model of categorization decisions under uncertainty. Psychological review, 105(2):280.

Fleming, S. M., Massoni, S., Gajdos, T., and Vergnaud, J.-C. (2016). Metacognition about the past and future: quantifying common and distinct influences on prospective and retrospective judgments of self-performance. Neuroscience of Consciousness, 2016(1):niw018.

Furnham, A. (1986). The robustness of the recency effect: Studies using legal evidence. The Journal of General Psychology, 113(4):351-357.

Garcia, T. and Massoni, S. (2017). Aiming to choose correctly or to choose wisely? the optimality-accuracy trade-off in decisions under uncertainty. GATE WP 1714.

Green, D. and Swets, J. (1966). Signal detection theory and psychophysics. Los Altos, CA: 
Peninsula.

Han, S. and Dobbins, I. G. (2008). Examining recognition criterion rigidity during testing using a biased-feedback technique: Evidence for adaptive criterion learning. Memory $\mathcal{G}^{3}$ Cognition, 36(4):703-715.

Hogarth, R. M. and Einhorn, H. J. (1992). Order effects in belief updating: The beliefadjustment model. Cognitive psychology, 24(1):1-55.

Jarvik, M. E. (1951). Probability learning and a negative recency effect in the serial anticipation of alternative symbols. Journal of experimental psychology, 41(4):291.

Jones, M. and Sieck, W. R. (2003). Learning myopia: an adaptive recency effect in category learning. Journal of Experimental Psychology: Learning, Memory, and Cognition, 29(4):626.

Maddox, W. T. and Bohil, C. J. (1998). Base-rate and payoff effects in multidimensional perceptual categorization. Journal of Experimental Psychology: Learning, Memory, and Cognition, 24(6):1459.

Maddox, W. T. and Bohil, C. J. (2004). Probability matching, accuracy maximization, and a test of the optimal classifiers independence assumption in perceptual categorization. Perception \& psychophysics, 66(1):104-118.

Maddox, W. T. and Bohil, C. J. (2005). Optimal classifier feedback improves cost-benefit but not base-rate decision criterion learning in perceptual categorization. Memory $\mathcal{E}$ Cognition, 33(2):303-319.

Maddox, W. T., Bohil, C. J., and Dodd, J. L. (2003). Linear transformations of the payoff matrix and decision criterion learning in perceptual categorization. Journal of Experimental Psychology: Learning, Memory, and Cognition, 29(6):1174.

Maddox, W. T. and Dodd, J. L. (2001). On the relation between base-rate and cost-benefit learning in simulated medical diagnosis. Journal of Experimental Psychology: Learning, Memory, and Cognition, 27(6):1367.

Mantonakis, A., Rodero, P., Lesschaeve, I., and Hastie, R. (2009). Order in choice: Effects 
of serial position on preferences. Psychological Science, 20(11):1309-1312.

Massoni, S., Gajdos, T., and Vergnaud, J.-C. (2014). Confidence measurement in the light of signal detection theory. Frontiers in psychology, 5:1455.

Milkman, K. L., Chugh, D., and Bazerman, M. H. (2009). How can decision making be improved? Perspectives on psychological science, 4(4):379-383.

Murdock Jr, B. B. (1962). The serial position effect of free recall. Journal of experimental psychology, 64(5):482-488.

Pitz, G. F. and Downing, L. (1967). Optimal behavior in a decision-making task as a function of instructions and payoffs. Journal of experimental psychology, 73(4, Pt.1):549555.

Towse, J. and Hitch, G. (1995). Is there a relationship between task demand and storage space in tests of working memory capacity? The Quarterly Journal of Experimental Psychology, 48(1):108-124.

Trotman, K. and Wright, A. (2000). Order effects and recency: where do we go from here? Accounting \& Finance, 40(2):169-182.

Tzeng, O. J. (1973). Positive recency effect in a delayed free recall. Journal of Verbal Learning and Verbal Behavior, 12(4):436-439.

Unsworth, N. and Engle, R. W. (2007). On the division of short-term and working memory: an examination of simple and complex span and their relation to higher order abilities. Psychological bulletin, 133(6):1038-1066. 


\section{Supplementary Information}

\section{Procedure}

This section details the stimuli and the feedback system. The study has been programmed using MATLAB with the Psychophysics Toolbox version 3 (Brainard, 1997) and has been achieved on computers of resolution $1920 \times 1080$. Subjects were enrolled using ORSEE (Greiner, 2015).

\subsection{Stimuli}

Calibration - The stimuli difficulty was given by the difference in numbers of dots between the two circles (hereafter referred as $c$ ). The calibration of the difficulty of the task was done using a psychophysic staircase at the subject level in order to control for individual heterogeneity of vision abilities (Levitt, 1971). This one-up two-down staircase consists in removing one dot after two consecutive correct answers and adding one dot after one failure. The calibration ended when subjects had achieved 35 reversals in the staircase (a reversal means a change in the $c$ 's value). The number of dots difference $c$ used in the experiment was computed as the mean dots number across the two last reversals of the staircase. In this non-incentivized calibration period, subjects received only the stimuli information and had to determine which circle contains the most of dots. Feedback on decision accuracy was provided after each decision. The targeted accuracy rate was $71 \%$. This level was chosen for subjects to outperform random guesses (relevance of stimuli) and to avoid being too close to perfect discrimination (relevance of the payoff matrix).

Stimuli generation - For a same level of calibration, we presented the exact same set of 
stimuli to participants in each block by difficulty level. Stimuli were randomly generated prior to the experiment under constraints concerning dots spacing. All dots were of the same size (diameter $0.4^{\circ}$ ) and the average distance between dots was kept constant. They appeared at random positions inside two outline circles (diameter $5.1^{\circ}$ ). Before and after dots appearance, fixation crosses were displayed at their centers at eccentricities of $\pm 8.9^{\circ}$.

\subsection{Feedback}

The feedback system was composed of trial feedback and aggregated feedback. We aimed not to influence participants to consider more stimuli or payoff matrices. Thus, we gave them a feedback on stimuli - if their decisions were correct or incorrect - and a feedback on the payoff matrix - number of points earned for the decision. Additionally to these trialby-trial feedback, subjects received every 12 decisions two aggregated feedback. First, we showed their accuracy rate for the last 12 decisions. Second, we provided an aggregated point feedback indicating how close they were from perfect performances. Formally the aggregated point feedback (apf) was given by the following formula:

$$
a p f=\frac{x-y}{z-y}
$$

With $x$ the actual number of points earned, $y$ the number of points earned if all answers would have been incorrect, $z$ the number of points earned if all answers would have been correct. We provide subjects with these aggregated feedback i) to induce a matching between subjective beliefs and actual performances, ii) to allow non-decision periods within blocks.

\subsection{Model specification: Gaussian equal-variance SDT}

This section presents how we specify our model as a Gaussian equal-variance SDT. We start by modelling the perceptual part of the decision based on our 2AFC stimuli.Then, we use the payoffs provided by the payoff matrix to distinguish the four different outcomes 
of SDT.

The perceptual task was a $2 \mathrm{AFC}$ task in which subjects faced two circles with dots inside and aimed to identify the circle with the more dots. The SDT theory specifies that evidence received from each circle follows a normal distribution. For a larger number of dots, the amount of evidence is in average larger. The variance parameters represent subjects' visual capacity to evaluate stimuli. The difference in dots' number being relatively small, we make the usual assumption that both variance parameters are equal.

We note $X_{s}$ the perception of the circle with the most dots and $X_{n}$ the perception of the circle with the least dots:

$$
X_{n} \sim \mathcal{N}\left(\mu_{n}, \sigma^{2}\right) \text { and } X_{s} \sim \mathcal{N}\left(\mu_{s}, \sigma^{2}\right)
$$

with $\mu_{n}<\mu_{s}$.

Additionally to containing more or less dots, each answer is either associated with high or low payoffs when correct. To include this dimension, we note $X_{H}$ the perception of the circle associated with higher rewards and $X_{L}$ the perception of the circle associated with lower rewards. We note $e$ the perceived difference between these two circles:

$e=X_{H}-X_{L}= \begin{cases}X_{s}-X_{n} \sim \mathcal{N}\left(\mu_{s}-\mu_{n}, 2 \times \sigma^{2}\right) & \text { if the highly rewarded circle contains more dots } \\ X_{n}-X_{s} \sim \mathcal{N}\left(\mu_{n}-\mu_{s}, 2 \times \sigma^{2}\right) & \text { if the highly rewarded circle contains less dots }\end{cases}$

The model can be specified as a Gaussian equal-variance Signal detection task by defining "signal" the event "the highly rewarded circle contains more dots" and "noise" the opposite event "the highly rewarded circle contains less dots". In fact, $e$ can be written as:

$$
e \sim \begin{cases}\mathcal{N}(0,1) & \text { if "noise" } \\ \mathcal{N}\left(d^{\prime}, 1\right) & \text { if "signal" }\end{cases}
$$


with $d^{\prime}=\frac{2 \times\left(\mu_{s}-\mu_{n}\right)}{\sqrt{2} \times \sigma}$ and $\epsilon=\frac{Y+\left(\mu_{s}-\mu_{n}\right)}{\sqrt{2} \times \sigma}$.

It is thus equivalent to the Gaussian equal variance SDT in its general specification. Under this framework, the four different outcomes are defined as:

- Hit: Reporting the highly rewarded circle when it contains more dots.

- Correct rejection: Reporting the lowly rewarded circle when it contains more dots.

- Miss: Reporting the lowly rewarded circle when it contains less dots.

- False alarm: Reporting the highly rewarded circle when it contains less dots.

Based on $e$, information extracted from stimuli is given by the likelihood function:

$$
\mathcal{L}(e)=\frac{\mathbb{P}(e \mid S)}{\mathbb{P}(e \mid N)}
$$

The decision strategy $\beta$ is summarized by the minimal value of the likelihood function required to answer signal (reporting the circle associated with higher rewards). This value has to be interpreted as follows: an individual with a decision strategy $\beta$ reports the circle associated with the higher rewards when it is (at least) $\beta$ times more likely than the other circle to contain more dots.

The optimal decision strategy is given by answering signal if and only if:

$$
\mathcal{L}(e)>\frac{\Pi(\text { correctrejection })-\Pi(\text { false alarm })}{\Pi(\text { hit })-\Pi(\text { miss })} \times \frac{\mathbb{P}(N)}{\mathbb{P}(S)} \equiv \beta^{*}
$$

Where $\Pi$ refers to the payoff function. In our study subjects are acknowledged that we implement equal base rates i.e. signal and noise are equally likely $\left(\mathbb{P}(S)=\mathbb{P}(N)=\frac{1}{2}\right)$.

To conclude this section on our framework, we address the question of potential position bias. Position bias are bias to choose either "Left" or "Right". Our modelisation is orthogonal to spatial location as it takes as reference the circle with the more dots and not its position. However, it does not affect our results as the experiment is designed to 
cancel out these potential bias as target circles and highly rewarded circles are balanced across sides.

\section{SDT estimation}

This section details how we compute the descriptive statistics and how we estimate SDT parameters.

Descriptive statistics - The hit rate $(H R)$ is equal to the number of hits $\left(n_{H}\right)$ divided by the number of signal trials $\left(n_{s}\right)$. Likewise, false alarm rate $(F A R)$ is equal to the number of false alarms $\left(n_{F A}\right)$ divided by the number of noise trials $\left(n_{n}\right)$

$$
H R=\frac{n_{H}}{n_{s}} \text { and } F A R=\frac{n_{F A}}{n_{n}}
$$

Estimations - To estimate a couple $\left(\hat{d}^{\prime}, \hat{\beta}\right)$ for each information order:

(i) We express the probabilities of hit $\left(P_{H}\right)$ and false alarm $\left(P_{F A}\right)$ as functions of $\left(d^{\prime}, \beta\right)$ :

$$
\begin{gathered}
P_{H}\left(d^{\prime}, \beta\right)=1-\phi\left(\frac{\log (\beta)}{d^{\prime}}-d^{\prime} / 2\right) \\
P_{F A}\left(d^{\prime}, \beta\right)=1-\phi\left(\frac{\log (\beta)}{d^{\prime}}+d^{\prime} / 2\right)
\end{gathered}
$$

Where $\phi$ refers to the cumulative distribution function of the standard normal distribution.

(ii) We compute the probability that, for a couple $\left(P_{H}, P_{F A}\right)$, we obtain the observed number of hits and false alarms using binomial distributions.

$$
\begin{gathered}
f\left(n_{H} ; n_{s} ; P_{H}\right)=\left(\begin{array}{c}
n_{s} \\
n_{H}
\end{array}\right)\left(P_{H}\right)^{n_{H}}\left(1-P_{H}\right)^{n_{s}-n_{H}} \\
f\left(n_{F A} ; n_{n} ; P_{F A}\right)=\left(\begin{array}{c}
n_{n} \\
n_{F A}
\end{array}\right)\left(P_{F A}\right)^{n_{F A}}\left(1-P_{F A}\right)^{n_{n}-n_{F A}}
\end{gathered}
$$

(iii) We compute the couple $\left(d^{\prime}, \beta\right)$ that jointly maximizes $f\left(n_{H} ; n_{s} ; P_{H}\right)$ and $f\left(n_{F A} ; n_{n} ; P_{F A}\right)$ 
by maximum likelihood:

$$
\left(\hat{d}^{\prime}, \hat{\beta}\right)=\operatorname{argmax}\left(\log \left(f\left(n_{H} ; n_{s} ; P_{H}\left(d^{\prime}, \beta\right)\right)\right)+\log \left(f\left(n_{F A} ; n_{n} ; P_{F A}\left(d^{\prime}, \beta\right)\right)\right)\right)
$$

We use a similar approach to compute one discrimination and three matrix-specific decision criteria $\left(\hat{d}^{\prime}, \hat{\beta}_{12}, \hat{\beta}_{17}, \hat{\beta}_{21}\right)$ for each information order:

(i) We compute three couples $\left(P_{H}, P_{F A}\right)$ :

$$
P_{H}\left(d^{\prime}, \beta_{12}\right), P_{F A}\left(d^{\prime}, \beta_{12}\right), P_{H}\left(d^{\prime}, \beta_{17}\right), P_{F A}\left(d^{\prime}, \beta_{17}\right), P_{H}\left(d^{\prime}, \beta_{21}\right) \text { and } P_{F A}\left(d^{\prime}, \beta_{21}\right) \text {. }
$$

(ii) We compute the associated binomial distributions based on the number of hits and false alarms per matrix.

(iii) We compute the vector $\left(\hat{d}^{\prime}, \hat{\beta}_{12}, \hat{\beta}_{17}, \hat{\beta}_{21}\right)$ that jointly maximizes the binomial distributions by maximum likelihood.

\section{Descriptive Statistics}

Table 1 describes subjects' hit rates and false alarm rates for each payoff matrix in each treatment. It also gives these rates at a treatment level (referred as "All").

The order effect obtained in the paper is driven mainly by the false alarm rate. In fact, the hit rate of both treatments is not different $\left[t(57)=-0.8967, p=0.374, d_{z}=0.118\right]$ while the false alarm rate is higher in the SF treatment compared to the $\mathrm{PF}$ treatment $\left[t(57)=-2.886, p=<0.0055, d_{z}=0.379\right]$.

The matrix effect is salient for both hit rates $\left[F(2,114)=4.758, p=0.0104, \eta_{p}^{2}=0.091\right]$ and false alarm rates $\left[F(2,114)=5.694, p=0.0044, \eta_{p}^{2}=\right] . \quad M_{21}$ is the matrix inducing this effect. With $M_{21}$ subjects achieve more hits than with $M_{17}[q=3.076, p=0.0059$, $\left.d_{z}=0.404\right]$ and more false alarms than with $M_{12}\left[q=3.277, p=0.0032, d_{z}=0.430\right]$. Additionally, we also observe slightly more false alarms with $M_{17}$ than with $M_{12}[q=2.337$, $\left.p=0.051, d_{z}=0.307\right]$. 


\begin{tabular}{|c|c|c|c|c|c|c|}
\hline $\begin{array}{l}\text { Statistic } \\
\mathrm{n}=58\end{array}$ & Treatment & Payoff matrix & $\begin{array}{c}M \\
(\%) \\
\end{array}$ & $S D$ & $\begin{array}{l}\text { Min } \\
(\%)\end{array}$ & $\begin{array}{l}\text { Max } \\
(\%)\end{array}$ \\
\hline \multirow[t]{8}{*}{ Hit Rate } & $P F$ & $M_{12}$ & 72.56 & 9.17 & 50.00 & 91.67 \\
\hline & & $M_{17}$ & 71.05 & 14.32 & 37.50 & 100.00 \\
\hline & & $M_{21}$ & 74.57 & 10.37 & 50.00 & 95.83 \\
\hline & & $A l l$ & 72.73 & 8.49 & 52.78 & 94.44 \\
\hline & $S F$ & $M_{12}$ & 74.21 & 9.32 & 54.17 & 95.83 \\
\hline & & $M_{17}$ & 70.69 & 15.69 & 29.17 & 100.00 \\
\hline & & $M_{21}$ & 76.08 & 9.12 & 54.17 & 95.83 \\
\hline & & $A l l$ & 73.66 & 8.62 & 52.78 & 95.83 \\
\hline \multirow[t]{8}{*}{ False Alarm Rate } & $P F$ & $M_{12}$ & 33.91 & 12.74 & 8.33 & 62.50 \\
\hline & & $M_{17}$ & 35.56 & 15.06 & 4.17 & 79.17 \\
\hline & & $M_{21}$ & 36.85 & 16.01 & 8.33 & 79.17 \\
\hline & & All & 35.44 & 13.00 & 11.11 & 70.83 \\
\hline & $S F$ & $M_{12}$ & 35.78 & 14.24 & 4.17 & 87.50 \\
\hline & & $M_{17}$ & 40.01 & 16.18 & 12.50 & 91.67 \\
\hline & & $M_{21}$ & 41.09 & 15.97 & 8.33 & 91.67 \\
\hline & & All & 38.96 & 13.56 & 15.28 & 90.28 \\
\hline
\end{tabular}

Table 1: Mean performances in terms of hit rate and false alarm rate. Treatments are Payoff-First (PF) and Stimuli-First (SF). $M_{12}, M_{17}$ and $M_{21}$ respectively correspond to "Low", "Intermediate" and "High" payoff contrasts. 


\section{Robustness test of criterion estimation}

In the paper, we present an estimation of one couple $\left(\hat{d}^{\prime}, \hat{\beta}\right)$ for each information order at a participant level (Estimation (1)) and another estimation of one discrimination and three matrix-specific decision criteria $\left(\hat{d}^{\prime}, \hat{\beta}_{12}, \hat{\beta}_{17}, \hat{\beta}_{21}\right)$ for each information order at a subject level (Estimation (2)). Estimation (1) leads to slightly highest $\hat{d}^{\prime}(1.39 \%$ in average and a maximum of $6.08 \%, t(115)=-11.672, p<0.0001)$ compared to Estimation $(2)$. Another method consists in a two-step estimation. Estimate $d^{\prime}$ as in Estimation (1) then estimate the three matrix-specific decision criteria using $d^{\prime}$ previously estimated.

The choice could impact Result 4. This section produces results obtained with this second methodology and concludes that this result is robust to the methodology change.

The repeated ANOVA rejects the hypothesis that matrix-specific mean decision strategies are equal $\left[F(2,114)=3.168, p=0.0458, \eta_{p}^{2}=0.05265\right]$. The Tukey's test reveals that the only significant difference is between matrix $M_{21}$ and $M_{12}[q=-2.505, p=$ $\left.0.0329, d_{z}=0.329\right]$.

\section{Instruction}

\section{Task}

In this experiment, you will play a total of 4 blocks of 72 trials. In each trial, you will receive two pieces of information.

- Stimuli: two circles containing different number of dots. Each circle has a $50 \%$ chance of containing more dots than the other :

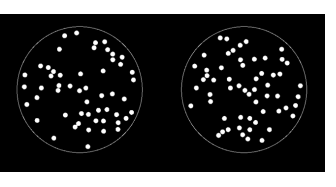

- Payoff Table: number of points earned according to the outcome of your decision. The Payoff Table will be randomly selected among one of the six following tables. 


\begin{tabular}{l|c|c} 
Decision & Left & Right \\
\hline Correct & 12 & 10 \\
Incorrect & 9 & 9
\end{tabular}

\begin{tabular}{l|c|c} 
Decision & Left & Right \\
\hline Correct & 21 & 1 \\
Incorrect & -9 & -9
\end{tabular}

\begin{tabular}{l|c|c} 
Decision & Left & Right \\
\hline Correct & 17 & 5 \\
Incorrect & -1 & -1
\end{tabular}

\begin{tabular}{l|c|c} 
Decision & Left & Right \\
\hline Correct & 10 & 12 \\
Incorrect & 9 & 9
\end{tabular}

\begin{tabular}{l|c|c} 
Decision & Left & Right \\
\hline Correct & 1 & 21 \\
Incorrect & -9 & -9
\end{tabular}

\begin{tabular}{l|c|c} 
Decision & Left & Right \\
\hline Correct & 5 & 17 \\
Incorrect & -1 & -1
\end{tabular}

\section{Decision}

In each trial, once you received both information, you need to select one of the circles of the stimuli.

- If the selected circle is the one with the most dots, it is correct. Otherwise, it is incorrect.

- According to your decision and the Payoff Table, different number of points are associated to correct and incorrect decisions.

- To select the left circle, press the key $\leftarrow$ on your keyboard.

- To select the right circle, press the key $\rightarrow$ on your keyboard.

\section{Blocks}

- From one block to another, the information is not displayed in the same order:

- In "Type A blocks", you receive first the Payoff Table then the Stimuli previously to decision.

- In "Type 1 blocks", you receive first the Stimuli then the Payoff Table previously to decision.

- You will play a total of 4 blocks alternating between the two types.

\section{Feedback}

- After each trial you will know if you were correct or not and the number of points earned. 
- Every 12 trials, you will receive:

- An aggregated accuracy feedback: the percentage of correct answers in the last 12 decisions.

- An aggregated point feedback: how close you were from the highest number of points compared to the lowest number of points in the last 12 decisions.

\section{Earnings}

Additionally to the AUD 5 of show-up fee, your variable payment will be based on :

- 6 trials randomly selected in each of 4 blocks.

- Your variable payment is the sum of all points accumulated during the 24 selected decisions (equal proportion of each Payoff Table).

- The conversion rate between points and AUD is:

$$
10 \text { points }=A U D 1
$$

\section{References}

Brainard, D. H. (1997). The psychophysics toolbox. Spatial vision, 10:433-436.

Greiner, B. (2015). Subject pool recruitment procedures: organizing experiments with orsee. Journal of the Economic Science Association, 1(1):114-125.

Levitt, H. (1971). Transformed up-down methods in psychoacoustics. The Journal of the Acoustical society of America, 49(2B):467-477. 